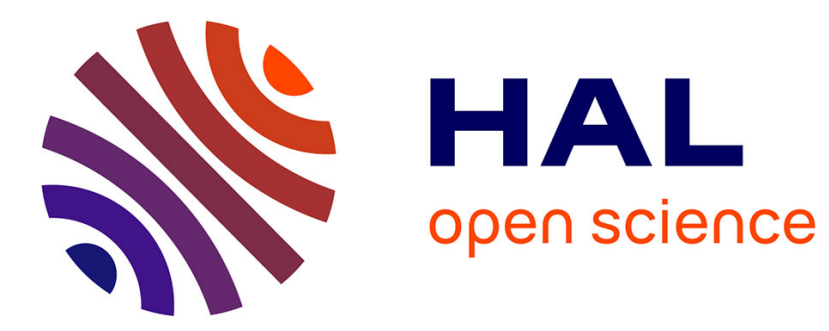

\title{
Electrical properties of magnetic nanocontact devices computed using finite-element simulations
}

\author{
S. Petit-Watelot, R M Otxoa, M Manfrini
}

\section{To cite this version:}

S. Petit-Watelot, R M Otxoa, M Manfrini. Electrical properties of magnetic nanocontact devices computed using finite-element simulations. Applied Physics Letters, 2012, 100, pp.83507 - 153906. $10.1063 / 1.3687915$. hal-01456515

\section{HAL Id: hal-01456515 \\ https://hal.univ-lorraine.fr/hal-01456515}

Submitted on 5 Feb 2017

HAL is a multi-disciplinary open access archive for the deposit and dissemination of scientific research documents, whether they are published or not. The documents may come from teaching and research institutions in France or abroad, or from public or private research centers.
L'archive ouverte pluridisciplinaire HAL, est destinée au dépôt et à la diffusion de documents scientifiques de niveau recherche, publiés ou non, émanant des établissements d'enseignement et de recherche français ou étrangers, des laboratoires publics ou privés.

\section{()ㅜ) $\Theta$}

Distributed under a Creative Commons Attribution - NoDerivatives| 4.0 International 


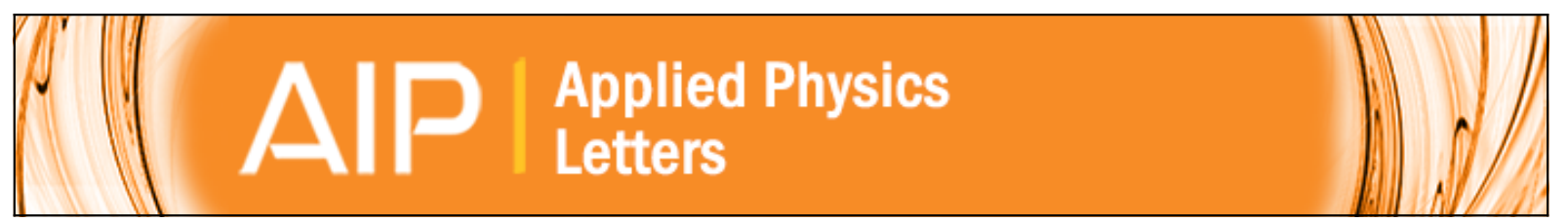

\section{Electrical properties of magnetic nanocontact devices computed using finite-element simulations}

S. Petit-Watelot, R. M. Otxoa, and M. Manfrini

Citation: Applied Physics Letters 100, 083507 (2012); doi: 10.1063/1.3687915

View online: http://dx.doi.org/10.1063/1.3687915

View Table of Contents: http://scitation.aip.org/content/aip/journal/apl/100/8?ver=pdfcov

Published by the AIP Publishing

\section{Articles you may be interested in}

Skyrmion stability in nanocontact spin-transfer oscillators

AlP Advances 5, 097126 (2015); 10.1063/1.4930904

Non-stationary excitation of two localized spin-wave modes in a nano-contact spin torque oscillator J. Appl. Phys. 114, 153906 (2013); 10.1063/1.4825065

Enhancement of microwave oscillation under angled in-plane magnetic field in ferromagnetic nano-contact spinvalves

Appl. Phys. Lett. 99, 092507 (2011); 10.1063/1.3619835

Nanocontact spin-transfer oscillators based on perpendicular anisotropy in the free layer Appl. Phys. Lett. 91, 162506 (2007); 10.1063/1.2797967

Self-consistent simulation of quantum transport and magnetization dynamics in spin-torque based devices Appl. Phys. Lett. 89, 153504 (2006); 10.1063/1.2359292

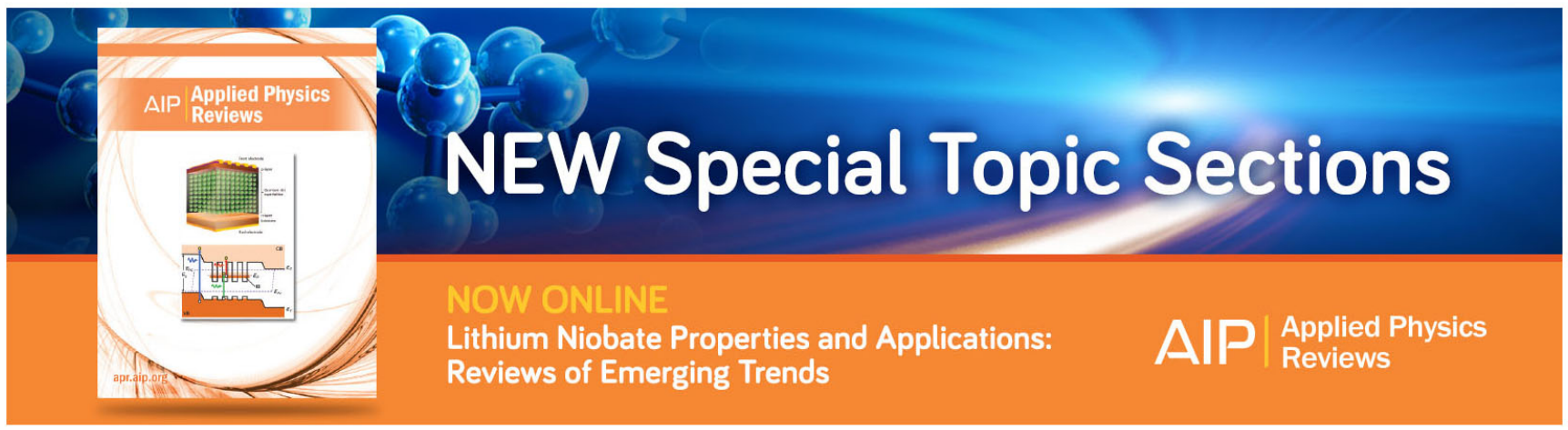




\title{
Electrical properties of magnetic nanocontact devices computed using finite-element simulations
}

\author{
S. Petit-Watelot, ${ }^{1,2, a)}$ R. M. Otxoa, ${ }^{1,2}$ and M. Manfrini ${ }^{3,4}$ \\ ${ }^{1}$ Institut d' Electronique Fondamentale, Université Paris-Sud, 91405 Orsay, France \\ ${ }^{2} U M R$ 8622, CNRS, 91405 Orsay, France \\ ${ }^{3}$ IMEC, Kapeldreef 75, B-3001 Leuven, Belgium \\ ${ }^{4}$ Laboratorium voor Vaste-Stoffysica en Magnetisme, K. U. Leuven, Celestijnenlaan 200 D, \\ B-3001 Leuven, Belgium
}

(Received 9 December 2011; accepted 30 January 2012; published online 23 February 2012)

\begin{abstract}
We compute the electrical current flow and associated Oersted fields in magnetic nanocontact (NC) systems with finite-element simulations. Underneath the nanocontact, the current distribution is found to be mainly perpendicular to the film plane, while the flow is essentially in the film plane outside the nanocontact region. For circular nanocontacts, we provide criteria for which the cylindrical approximation remains valid for electrical flow in asymmetric rectangular devices. The nature of the current distribution, and the induced field, is a key to understanding spin-torque driven magnetization dynamics in such systems. (C) 2012 American Institute of Physics. [doi:10.1063/1.3687915]
\end{abstract}

The possibility to control magnetization dynamics with a spin polarized current, through the spin transfer effect, ${ }^{1,2}$ has allowed the conception of numerous new spintronic devices such as magnetic nonvolatile memories and nanooscillators. In such devices, different kinds of magnetization dynamics can be induced, depending on the sample geometry, material parameters, and experimental conditions. ${ }^{3,4}$ Because appreciable spin transfer torques require large current densities, nanocontacts are often used to deliver currents to magnetic multilayer structures. This geometry allows for a large variety of magnetization oscillation modes ${ }^{5-7}$ and the possibility to synchronize the dynamics induced by several point contacts attached to the same multilayer. ${ }^{8}$

A key aspect governing the magnetization dynamics is the profile of the current flow and the Oersted field associated with it. ${ }^{9-12}$ In general, the real geometry of NC devices does not allow for a quantitative description to be possible by simple geometric considerations, ${ }^{13,14}$ even when cylindrical symmetry is present. ${ }^{15,16}$ Furthermore, the current profile also depends strongly on the different material conductivities in the material stack.

In this paper, we identify the relevant materials and geometrical parameters that govern the quantitative distribution of the three components of the current density and induced Oersted field NC devices. ${ }^{7}$ We first investigate how the cylindrical symmetry of a $\mathrm{NC}$ is broken when considering real boundary conditions using full 3D simulations. We then define criteria to estimate the deviation from the cylindrical approximation. In the second part, we give a detailed description of the current distribution and induced field close to the $\mathrm{NC}$ and discuss the effects of the materials and shape of the NC.

The device studied is a circular NC formed through a $50 \mathrm{~nm} \mathrm{SiO}_{2}$ layer, supplying current to an extended spinvalve stack $17.2 \mu \mathrm{m}$ wide and $27 \mu \mathrm{m}$ long. The shape of the

\footnotetext{
${ }^{\text {a) }}$ Author to whom correspondence should be addressed. Electronic mail: sebastien.petit-watelot@u-psud.fr.
}

$\mathrm{NC}$ is a truncated cone, with radius $r_{b}=60 \mathrm{~nm}$ at its bottom and $r_{t}=110$ at the top, top-contacted with a rectangular stripe. The current is extracted by a ground contact made on top of the spin-valve through a $10 \mu \mathrm{m}$ by $17 \mu \mathrm{m}$ opening, located at $11 \mu \mathrm{m}$ away from the NC. The composition of the stack, from top to bottom, is (thickness in nanometers): $\mathrm{Pt}$ $3 / \mathrm{Ni}_{80} \quad \mathrm{Fe}_{20} 5 / \mathrm{Cu} 3.5 / \mathrm{Co}_{90} \mathrm{Fe}_{10}$ 4.5/IrMn 6/Seed 50. The magnetoresistive part corresponds to the exchanged pinned $\mathrm{Co}_{90} \mathrm{Fe}_{10}$ layer, the $\mathrm{Cu}$ spacer, and the Permalloy $\left(\mathrm{Ni}_{80}\right.$ $\left.\mathrm{Fe}_{20}\right)$ free layer. The seed layer is a multilayered $\mathrm{Ta} / \mathrm{Cu}$ structure which is treated as a uniform material.

A cylindrical approximation for the $\mathrm{NC}$ is often used for analytical calculations and simulations in order to minimize calculation time and power. Unfortunately for real devices, the finite dimensions of the spin-valve structure and the way contacts are made break this symmetry. We investigated how realistic boundary conditions affect the cylindrical symmetry of the $\mathrm{NC}$ using full 3D finite-element simulations $\left(\mathrm{Comsol}^{\circledR}\right)$. The lateral output surface of the ground electrode is constrained to $0 \mathrm{~V}$ and the top electrode is biased such that the current density is normal to its lateral input surface. The incoming current density corresponds to a total current of $1 \mathrm{~mA}$. Without loss in generality, the spin-valve stack is assumed to have a uniform conductivity only in this part.

Figure 1 represents the voltage distribution in the plane of the spin-valve taken at a depth corresponding to the middle of the free layer. The equipotential lines are almost circular around the $\mathrm{NC}$ and become progressively parallel to the ground plane. Consequently, the current lines are almost radial near the NC, as for a vertical line source. Since magnetization dynamics takes place in a small area around the NC, where the amplitude of the current density remains significant, we will restrict our study to a circular region, with a radius of $2 \mu \mathrm{m}$, around the NC. Let us use polar coordinates $(r, \theta)$ centered on the NC revolution axes. Because the ground electrode ensures that the current is uniform far from the NC, a simple way to describe the real current close to the 


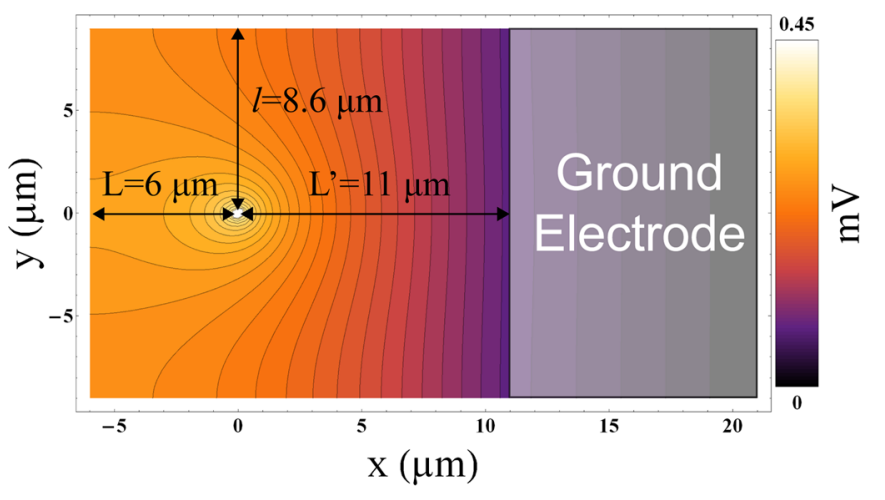

FIG. 1. (Color online) Voltage distribution in the plane of the spin-valve stack and associated equipotential lines. $(x=0, y=0)$ is taken at the center of the NC.

$\mathrm{NC}$ is to consider a pure cylindrical current flow $\left(\vec{J}_{\mathrm{CIP}}^{\mathrm{cyl}}\right)$ superimposed with an uniform in-plane current. The real inplane current density $\left(\vec{J}_{\mathrm{CIP}}^{\text {real }}\right)$ is a function of $(r, \theta)$ when $J_{\mathrm{CIP}}^{\mathrm{cyl}} \overrightarrow{e_{r}}$ is only $r$ dependent. As expected from this simple picture, the mean value of the CIP current amplitude on a circle with radius $r,\left\langle J_{\mathrm{CIP}}^{\mathrm{real}}(r, \theta)\right\rangle_{\theta}$, is equal to $J_{\mathrm{CIP}}^{\mathrm{cyl}}(r)$ (Inset Fig. 2). In order to estimate the deviation from the cylindrical symmetry in this area, we express $\vec{J}_{\mathrm{CIP}}^{\text {real }}$ in terms of its amplitude $J_{\mathrm{CIP}}^{\text {real }}(r, \theta)=J_{\mathrm{cyl}} \mathrm{CIP}(r)+\underset{\rightarrow r e a l}{\Delta}\left|J_{\mathrm{CIP}}\right|(r, \theta)$ and direction in the film plane $\phi(r, \theta)=\left\{\vec{J}_{C I P}^{\text {real }}, \vec{J}_{C I P}^{c y l}\right\}$. According to our description, $\Delta J_{\mathrm{CIP}}(r, \theta)$ is well approximated by $\Delta J_{\mathrm{CIP}}(r) \cos (\theta)$ and $\phi(r, \theta)$ by $\Delta \phi(r) \sin (\theta)$ for $r \ll l, L$, and $L^{\prime}$ (Fig. 1). $\Delta J_{\mathrm{CIP}}(r)$ and $\Delta \phi(r)$ are plotted in Fig. 2.

The difficulty involves evaluating the uniform contribution which is considered constant in the area of interest. For example, in the case of Fig. 1 , at the position $(-L, 0)$ from the NC, the uniform contribution should counterbalance the opposite radial current to ensure no current flows outside of the device. In this case, we verify that $\Delta J_{\mathrm{CIP}}(r) \approx r / L$. More generally, $\Delta J_{\mathrm{CIP}}(r)$ is proportional to $1 / \min \left\{L, L^{\prime}\right\}$. On the other hand, the boundary conditions force the current to be in the $x$ direction at the lateral edges, and from the simulation, we check that $\Delta \phi(r)$ is essentially linear with a slope equal to $\pi / 2 l$. These conditions can be used as a guide to estimate the deviation from a pure cylindrical system.

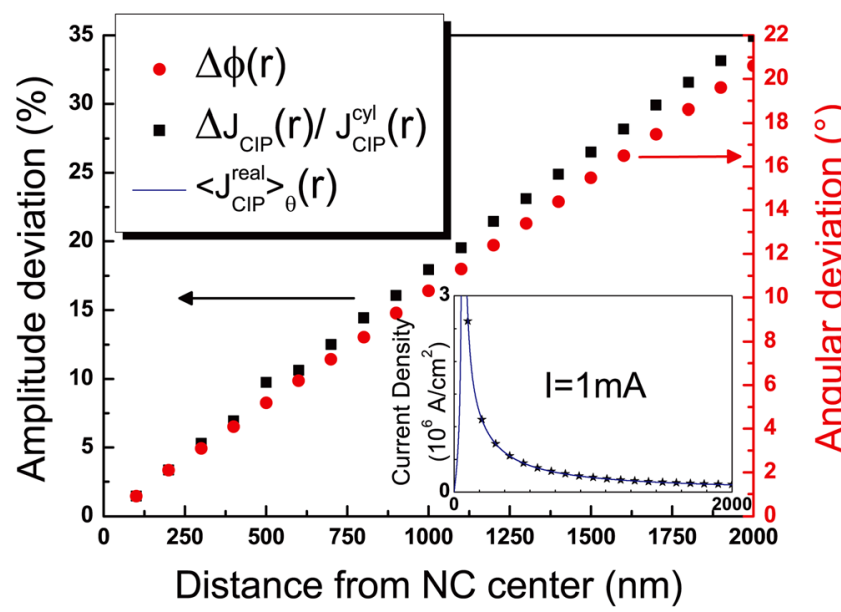

FIG. 2. (Color online) Angular deviation from the radial distribution (red points) and asymmetry. Inset: (stars) average in-plane current density and (blue curve) simulated current density using cylindrical symmetry.
In contrast, the stack geometry and the boundary conditions do not significantly affect the distribution of the current perpendicular to plane (CPP), provided $l, L$, and $L^{\prime}$ are kept greater than $\approx 2 \mu \mathrm{m}$. Since the Oersted field is almost only due to the CPP current, it is essentially orthoradial. However, the non-radial part of the CIP current induces a small additional uniform in-plane field along the $y$ direction. The amplitude and sign of this contribution depend on the depth in the stack and layers conductivities for a real spin-valve stack. In the case of uniform conductivity, its magnitude is about $8 \mathrm{~A} / \mathrm{m}$ at the free layer $(z=-5.5 \mathrm{~nm})$. This contribution causes a deviation from the cylindrical symmetry that increases almost linearly with $r$, reaching around $10 \%$ at $2 \mu \mathrm{m}$ from the $\mathrm{NC}$ in the film plane.

We conclude that the cylindrical approximation for the $\mathrm{NC}$ is good to first order, because the average in-plane current density decreases with a $1 / r$ slope, like the Oersted field far from the NC. At second order, it is possible to improve the accuracy of the cylindrical approximation using the corrections proposed below.

The cylindrical symmetry approximation reduces the computational overhead and allows us to compute the depth profile of the current in the stack. For this, we considered a lateral cut of the device from the center of the NC to a distance of $2 \mu \mathrm{m}$ without the top electrode. The $\mathrm{NC}$ is biased with a 1 $\mathrm{mA}$ current normal to the top of its cone, and the opposite edge of the cut is set to the ground potential. We now use the real spin-valve stack, with conductivities listed in Table I.

Figure 3 shows the current distribution in the sample taken at the middle of the free magnetic layer (Fig. 3(a)) and at the middle of the pinned layer (Fig. 3(b)). The CIP current is almost zero below the $\mathrm{NC}$ and reaches a maximum within $10 \mathrm{~nm}$ of the contact edge. Outside of the NC, the current is radial, decays inversely with the distance, and its distribution in the thickness is almost uniform in each layer. It follows that the different layers can be considered as parallel conductors, where $J_{\mathrm{CIP}}$ in each layer is simply proportional to the layer conductivity divided by the mean conductivity of the entire stack. The shape of the NC (varying $r_{t}$ between $r_{b}$ and $200 \mathrm{~nm}$ ) has no effect on the in-plane current distribution outside of the NC (not shown). However, it modifies, more or less depending on the layers conductivities, the distribution of CIP current within the top layers near the NC edge. For a cylindrical NC $\left(r_{t}=r_{b}\right)$, the current profile is mostly CPP at the interface between the contact and the stack, and a CIP component appears near at the edges of the NC as the current diffuses away from it. However, opening the cone angle of the $\mathrm{NC}\left(r_{t}>r_{b}\right)$ focalizes the incoming current in

TABLE I. Material conductivities used for the simulations. In parenthesis: thickness measured in the associated reference.

\begin{tabular}{lcc}
\hline \hline Material $(\mathrm{nm})$ & Conductivity $\left(10^{6} \mathrm{~S} / \mathrm{m}\right)$ & Reference \\
\hline $\mathrm{Au}(200)$ & 52.63 & 17 \\
$\mathrm{Pt}($ bulk) & 8.90 & 18 \\
$\mathrm{Ni}_{80} \mathrm{Fe}_{20}(20)$ & 3.85 & 19 \\
$\mathrm{Cu}(4.5)$ & 0.20 & 20 \\
$\mathrm{Co}_{90} \mathrm{Fe}_{10}(5)$ & 8.06 & 21 \\
$\mathrm{IrMn}(10)$ & 0.56 & 22 \\
Seed layer (50) & 7.00 & 18 \\
\hline \hline
\end{tabular}




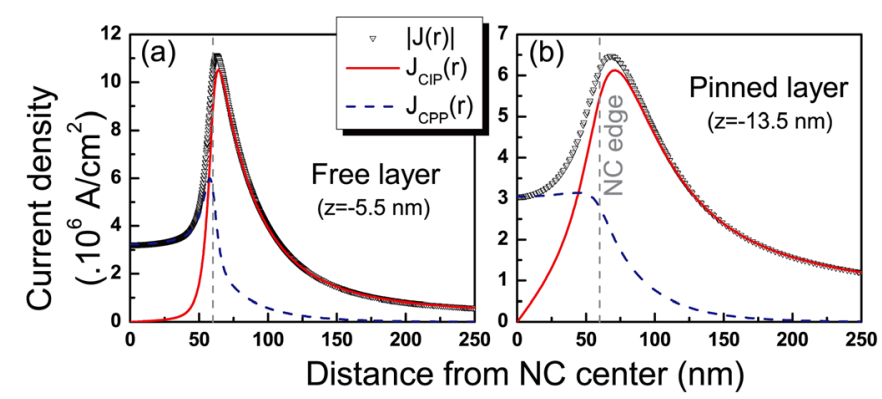

FIG. 3. (Color online) Distribution of the current density at the middle (a) of the permalloy layer (a) and (b) the pinned layer for a total $1 \mathrm{~mA}$ applied current. Dashed line (blue): perpendicular to plane component (CPP), solid line (red): in-plane current component (CIP), and triangles: total current density.

the stack, which leads to a reduction in the CIP component in the region just underneath the $\mathrm{NC}$ and an increase of the maximum at its edges.

In contrast, the CPP current distribution is more complex and no simple analytical approximations can be made. $J_{\mathrm{CPP}}$ is restricted below the NC, decaying extremely fast outside. Hence, it is mostly unaffected by the boundary conditions, but its radial distribution below the NC is not uniform, leading to a localized CPP torque. In the top layers, a maximum occurs near the NC edge (Fig. 3(a)). This maximum becomes less pronounced as we move deeper into the stack away from the top contact (Fig. 3(b)). The magnitude of $J_{\mathrm{CPP}}$ decreases almost linearly with the depth and reaches zero at the bottom of the stack. The rate of decay in each layer depends on its conductivity. The shape of the NC significantly alters the $J_{\mathrm{CPP}}$ distribution, depending on the conductivities of the constituent layers. For the conductivities used, there was almost no variation, but for a stack with uniform conductivity, it leads to appreciable changes. When the opening of the NC cone $\left(r_{b}\right)$ decreases, the CPP current amplitude increases below the center of the $\mathrm{NC}$ and decreases at its edges, leading to a more uniform distribution. This variation counterbalanced the variation of the CIP component already mentioned. Then, naturally as for the CIP current, the influence of the shape of $\mathrm{NC}$ on the $J_{\mathrm{CPP}}$ distribution is reduced if the cap layer possesses a large conductivity.

The nonuniform distribution of $J_{\mathrm{CPP}}$ results in a nontrivial spatial profile for the Oersted field, which is very different to that obtained assuming an infinite (or finite) current cylinder, $I_{\mathrm{CPP}}^{\mathrm{Py}} / 2 \pi a(r / a$ for $r<a, a / r$ for $r \geq a)$, as shown in Fig. 4(a). $I_{\mathrm{CPP}}^{\mathrm{Py}}$ is the integrated CPP current in the depth of the considered layer. One important difference from the infinite cylinder is that close to the $\mathrm{NC}$, the Oersted field decreases with the distance $r$ less rapidly than a $1 / r$ slope. This is partially due to the strong CIP current at the edges that induces an additional positive contribution in the top layers. Moreover, the amplitude and distribution of the Oersted field are sensitive to the conductivities of the different layers, as shown by the different distribution found for a stack with a uniform conductivity (Fig. 4(a)). Fig. 4(b) shows the distribution of the Oersted field inside the spin-valve stack. Its distribution in the different layers reflects the variation of the CPP current with respect the thickness. Hence, the thicker the magnetic part of the stack, the more non uniform the field will be.
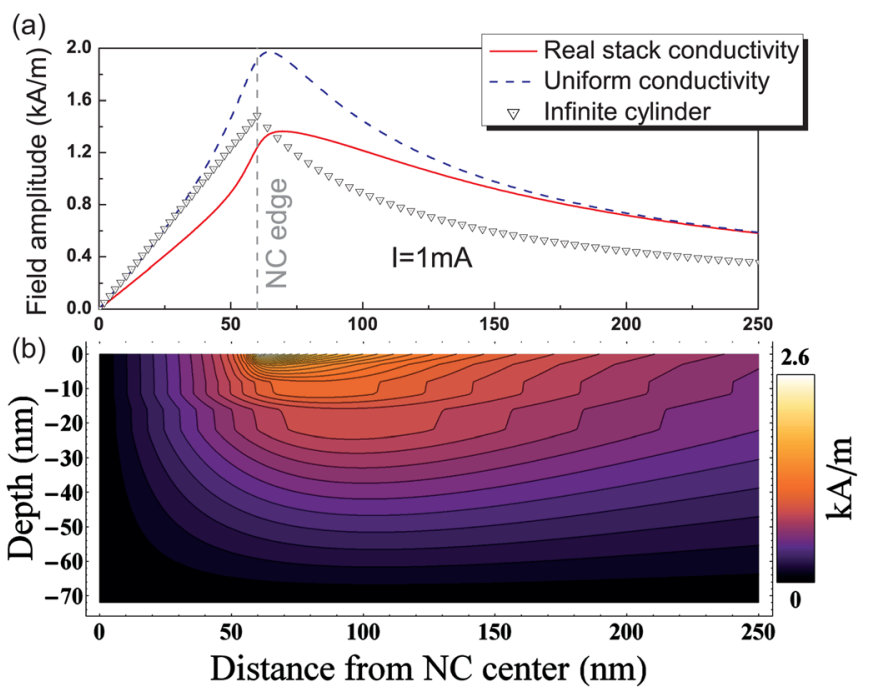

FIG. 4. (Color online) (a) Oersted field calculated in the middle of the free layer. Solid (red) curve: simulation with the real composition of the stack. Dashed (blue): simulation for a uniform conductivity. Triangle: calculations using the infinite cylindrical current approximation. (b) Distribution of the Oersted field inside the stack using the real material conductivities. Black lines: iso-field contours.

In summary, we have presented detailed finite-element simulations of the electrical properties of magnetic nanocontacts. We have shown that both CPP current and Oersted field are well described using a cylindrical approximation, in the region of interest for the magnetization dynamics. In contrast, the CIP current is more affected by the geometry. As a result, the CIP current can add a small uniform in-plane contribution to the Oersted field. We have defined some criteria for which the cylindrical symmetry of the NC can be used and proposed corrections to include the effect of the lateral contacts. By applying this method, a depth-resolved distribution of the current and Oersted field has been obtained. We find that the CPP current is nonuniform and largely limited to the NC region, leading to a localized CPP transfer torque. An important consequence of the complex current distribution involves the induced Oersted field, which decays slower than the inverse of the radial distance from the $\mathrm{NC}$.

The authors thank J.-V. Kim and T. Devolder for fruitful discussions and support. This work was partially supported by the European Commission, under Contract Nos. MRTNCT-2006-035327 SPINSWITCH and MRTN-CT-2008215368-2 SEMISPINNET, and the French National Research Agency (ANR), within the VOICE Project No. ANR-09-NANO-006.

${ }^{1}$ J. C. Slonczewski, J. Magn. Magn. Mater. 159, L1 (1996).

${ }^{2}$ L. Berger, Phys. Rev. B 54, 9353 (1996).

${ }^{3}$ F. J. Albert, N. C. Emley, E. B. Myers, D. C. Ralph, and R. A. Buhrman, Phys. Rev. Lett. 89, 226802 (2002).

${ }^{4}$ W. H. Rippard, M. R. Pufall, S. Kaka, S. E. Russek, and T. J. Silva, Phys. Rev. Lett. 92, 027201 (2004).

${ }^{5}$ A. Slavin and V. Tiberkevich, Phys. Rev. Lett. 95, 237201 (2005).

${ }^{6}$ M. R. Pufall, W. H. Rippard, M. L. Schneider, and S. E. Russek, Phys. Rev. B 75, 140404 (2007).

${ }^{7}$ Q. Mistral, M. van Kampen, G. Hrkac, J.-V. Kim, T. Devolder, P. Crozat, C. Chappert, L. Lagae, and T. Schrefl, Phys. Rev. Lett. 100, 257201 (2008).

${ }^{8}$ S. Kaka, M. R. Pufall, W. H. Rippard, T. J. Silva, S. E. Russek, and J. A. Katine, Nature 437, 389 (2005). 
${ }^{9}$ K. Ito, T. Devolder, C. Chappert, M. J. Carey, and J. A. Katine, J. Phys. D: Appl. Phys. 40, 1261 (2007).

${ }^{10}$ M. A. Hoefer, T. J. Silva, and M. D. Stiles, Phys. Rev. B 77, 144401 (2008).

${ }^{11}$ Y. Acremann, J. P. Strachan, V. Chembrolu, S. D. Andrews, T. Tyliszczak, J. A. Katine, M. J. Carey, B. M. Clemens, H. C. Siegmann, and J. Stãhr, Phys. Rev. Lett. 96, 217202 (2006).

${ }^{12}$ V. V. Uhlĩr, S. Pizzini, N. Rougemaille, V. Cros, E. Jiménez, L. Ranno, O. Fruchart, M. Urbánek, G. Gaudin, J. Camarero et al., Phys. Rev. B 83, 020406 (2011).

${ }^{13}$ G. Consolo, L. Torres, L. Lopez-Diaz, and B. Azzerboni, IEEE Trans. Magn. 43, 2827 (2007).

${ }^{14} \mathrm{R}$. Hertel, "Oersted fields and current density profiles in spin-torque driven magnetization dynamics - Finite element modelling of realistic geometries," e-prints, arXiv:0804.4010 (2008)
${ }^{15}$ D. V. Berkov and N. L. Gorn, Phys. Rev. B 80, 064409 (2009).

${ }^{16}$ E. Jaromirska, L. Lopez-Diaz, A. Ruotolo, J. Grollier, V. Cros, and D. Berkov, Phys. Rev. B 83, 094419 (2011).

${ }^{17}$ H. Kurt, W. Chiang, C. Ritz, K. Eid, W. Pratt, and J. Bass, J. Appl. Phys. 93, 7918 (2003).

${ }^{18}$ Handbook of Chemistry and Physics, edited by W. M. Haynes, 92nd ed. (CRS Press, London, 2011).

${ }^{19}$ S. D. Kim, J. J. Lee, S. H. Lim, and H. J. Kim, J. Appl. Phys. 85, 5992 (1999).

${ }^{20}$ W. Prater, E. Allen, W. Lee, M. Toney, J. Daniels, and J. Hedstrom, Appl. Phys. Lett. 84, 2518 (2004).

${ }^{21}$ H. Yuasa, M. Yoshikawa, Y. Kamiguchi, K. Koi, H. Iwasaki, M. Takagishi, and M. Sahashi, J. Appl. Phys. 92, 2646 (2002).

${ }^{22}$ M. Seigler, Appl. Phys. Lett. 80, 820 (2002). 